\title{
Coupled Finite and Boundary Element Methods for Vibro-Acoustic Interface Problems
}

\author{
Arno Kimeswenger, Olaf Steinbach, and Gerhard Unger
}

\section{Vibro-Acoustic Interface Problem}

As a vibro-acoustic interface model problem we consider a three-dimensional elastic body, e.g., a submarine, which is completely immersed in a full space acoustic region, e.g., water [5]. Other applications that we have in mind are the sound radiation of passenger car bodies, where the acoustic region is bounded, or of partially immersed bodies such as ships, where the acoustic region is a half space [2].

In this paper, we consider both a direct simulation of the interface problem by using a symmetric coupled finite and boundary element approach, and an eigenvalue analysis to determine the eigenmodes of the coupled system. The time-harmonic vibrating structure in $\Omega_{s}$ is modeled by the Navier equations in the frequency domain, while the acoustic fluid in the unbounded exterior domain $\Omega_{f}$ is described by the Helmholtz equation,

$$
-\rho_{s} \omega^{2} \mathbf{u}-\mu \Delta \mathbf{u}-(\lambda+\mu) \operatorname{grad} \operatorname{div} \mathbf{u}=\mathbf{f} \text { in } \Omega_{s}, \quad \kappa^{2} p+\Delta p=0 \text { in } \Omega_{f} .
$$

In (1), $\lambda$ and $\mu$ are the Lamé parameters, $\rho_{s}$ and $\rho_{f}$ are the densities of the structure and of the acoustic fluid, respectively, $\omega$ is the frequency, and $\kappa=\omega / c \in \mathbb{R}$ is the wave number. Note that $\Omega_{s} \subset \mathbb{R}^{3}$ is in general a bounded, multiple connected domain with an interior boundary $\Gamma_{I}=\bar{\Gamma}_{D} \cup \bar{\Gamma}_{N}, \Gamma_{D} \cap \Gamma_{N}=\emptyset$, see Fig. 1, where boundary conditions of Dirichlet and Neumann type are given,

$$
\mathbf{u}=\mathbf{g}_{D} \text { on } \Gamma_{D}, \quad T \mathbf{u}:=\lambda(\operatorname{div} \mathbf{u}) \mathbf{n}+2 \mu \frac{\partial}{\partial n} \mathbf{u}+\mu \mathbf{n} \times \operatorname{curl} \mathbf{u}=\mathbf{g}_{N} \text { on } \Gamma_{N} .
$$

Fig. 1 Computational domain and boundary conditions

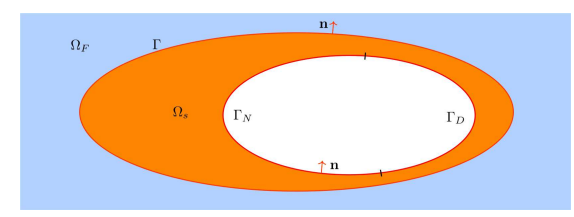

In addition to the partial differential equations (1) and the boundary conditions (2) we consider transmission conditions on $\Gamma=\bar{\Omega}_{S} \cap \bar{\Omega}_{f}$,

Arno Kimeswenger, Olaf Steinbach, Gerhard Unger

Institut für Numerische Mathematik, TU Graz, Steyrergasse 30, 8010 Graz, Austria

arno.kimeswenger@tugraz.at, o.steinbach@tugraz.at,

gerhard.ungeretugraz.at 


$$
T \mathbf{u}+p \mathbf{n}=\mathbf{0}, \quad \rho_{f} \omega^{2} \mathbf{u} \cdot \mathbf{n}=\mathbf{n} \cdot \nabla p \quad \text { on } \Gamma .
$$

Finally, $p$ has to satisfy a radiation condition at infinity,

$$
\lim _{r \rightarrow \infty} \int_{|x|=r}\left|\frac{\partial}{\partial n_{x}} p(x)-i \kappa p(x)\right|^{2} d s_{x}=0 .
$$

For complex wave numbers $\kappa \in \mathbb{C}$ with $\mathfrak{I}(\kappa)<0$, instead of (4) one has to use a radiation condition in terms of spherical Hankel functions in order to describe outgoing waves, see [12].

The aim of this paper is to derive and to discuss a symmetric coupled finite and boundary element formulation which is stable for almost all frequencies $\omega \in \mathbb{R}$, and to characterize all eigenfrequencies $\omega \in \mathbb{C}$ which imply non-trivial solutions of the homogeneous transmission problem (1)-(4), i.e. for $\mathbf{f}=\mathbf{0}, \mathbf{g}_{D}=\mathbf{0}, \mathbf{g}_{N}=\mathbf{0}$. In fact, in this case only one of the three following situations may appear [9]:

$i$. A real eigenfrequency $\omega \in \mathbb{R}$ implies $p=0$, and any non-trivial solution $\mathbf{u}$ is a so-called Jones mode satisfying $T \mathbf{u}=\mathbf{0}$ and $\mathbf{u} \cdot \mathbf{n}=0$ on $\Gamma$ [6].

ii. A complex value $\omega \in \mathbb{C}$ with $\mathfrak{I}(\omega)>0$ implies $\mathbf{u}=\mathbf{0}$ and $p=0$.

iii. If $\omega \in \mathbb{C} \backslash \mathbb{R}$ is an eigenfrequency, then $\mathfrak{I}(\omega)<0$.

In the low frequency regime one may consider an approximation of the Helmholtz equation in (1) by the Laplace equation, for related coupled finite and boundary element formulations, see [10].

\section{Coupled finite and boundary element methods}

The symmetric coupling [4] of finite and boundary elements for the transmission boundary value problem (1)-(4) relies on the standard variational formulation of the Navier equations in $\Omega_{s}$, and the use of the exterior Calderon projection of boundary integral equations [13] to describe the solution of the Helmholtz equation in $\Omega_{f}$. The resulting variational formulation is to find $\mathbf{u} \in\left[H^{1}\left(\Omega_{S}\right)\right]^{3}, \mathbf{u}=\mathbf{g}_{D}$ on $\Gamma_{D}$, such that

$$
\begin{gathered}
\int_{\Omega_{s}}[2 \mu e(\mathbf{u}): e(\mathbf{v})+\lambda \operatorname{div} \mathbf{u} \operatorname{div} \mathbf{v}] d x-\rho_{s} \omega^{2} \int_{\Omega_{s}} \mathbf{u} \cdot \mathbf{v} d x \\
-\rho_{f} \omega^{2}\left\langle V_{\kappa}[\mathbf{u} \cdot \mathbf{n}], \mathbf{v} \cdot \mathbf{n}\right\rangle_{\Gamma}+\left\langle\left(\frac{1}{2} I+K_{\kappa}\right) p, \mathbf{v} \cdot \mathbf{n}\right\rangle_{\Gamma}=\int_{\Omega_{s}} \mathbf{f} \cdot \mathbf{v} d x+\int_{\Gamma_{N}} \mathbf{g}_{N} \cdot \mathbf{v} d s_{x}
\end{gathered}
$$

is satisfied for all $\mathbf{v} \in\left[H^{1}\left(\Omega_{s}\right)\right]^{3}, \mathbf{v}=\mathbf{0}$ on $\Gamma_{D}$, where $p \in H^{1 / 2}(\Gamma)$ is a solution of the hypersingular boundary integral equation

$$
\frac{1}{\rho_{f} \omega^{2}} D_{\kappa} p+\left(\frac{1}{2} I+K_{\kappa}^{\prime}\right)[\mathbf{u} \cdot \mathbf{n}]=0 \quad \text { on } \Gamma .
$$


The boundary integral operators are defined as, for $x \in \Gamma$,

$$
\begin{aligned}
\left(V_{\kappa} q\right)(x) & =\int_{\Gamma} U_{\kappa}^{*}(x, y) q(y) d s_{y}, & \left(K_{\kappa} p\right)(x) & =\int_{\Gamma} \frac{\partial}{\partial n_{y}} U_{\kappa}^{*}(x, y) p(y) d s_{y} \\
\left(K_{\kappa}^{\prime} q\right)(x) & =\int_{\Gamma} \frac{\partial}{\partial n_{x}} U_{\kappa}^{*}(x, y) q(y) d s_{y}, & \left(D_{\kappa} p\right)(x) & =-\frac{\partial}{\partial n_{x}} \int_{\Gamma} \frac{\partial}{\partial n_{y}} U_{\kappa}^{*}(x, y) p(y) d s_{y}
\end{aligned}
$$

where the Helmholtz fundamental solution is

$$
U_{\kappa}^{*}(x, y)=\frac{1}{4 \pi} \frac{e^{i \kappa|x-y|}}{|x-y|} \quad \text { for } x, y \in \mathbb{R}^{3} .
$$

For the mapping properties of all boundary integral operators, see, for example, [13]. In particular, the hypersingular integral operator $D_{\kappa}: H^{1 / 2}(\Gamma) \rightarrow H^{-1 / 2}(\Gamma)$ is coercive and injective, if $\kappa^{2}$ is not an eigenvalue of the related interior Neumann eigenvalue problem of the Laplace operator in $\mathbb{R}^{3} \backslash \bar{\Omega}_{f}$. However, since we are using a direct approach we find $\left(\frac{1}{2} I+K_{\kappa}^{\prime}\right)[\mathbf{u} \cdot \mathbf{n}] \in \operatorname{Im} D_{\kappa}$ even in the case when $\kappa^{2}$ is an eigenvalue of the interior Neumann eigenvalue problem with a related eigensolution $p_{\kappa^{2} \mid \Gamma} \in H^{1 / 2}(\Gamma)[14]$, i.e.

$$
-\Delta p_{\kappa^{2}}=\kappa^{2} p_{\kappa^{2}} \text { in } \mathbb{R}^{3} \backslash \bar{\Omega}_{f}, \quad \frac{\partial}{\partial n} p_{\kappa^{2}}=0 \text { on } \Gamma .
$$

The general solution of the hypersingular boundary integral equation (6) is then given by

$$
p=-\rho_{f} \omega^{2} D_{\kappa}^{-1}\left(\frac{1}{2} I+K_{\kappa}^{\prime}\right)[\mathbf{u} \cdot \mathbf{n}]+\alpha p_{\kappa^{2}},
$$

where $D_{\kappa}^{-1}$ has to be understood as a pseudoinverse. Note that $\alpha \in \mathbb{R}$ is an arbitrary constant. However, when inserting the solution $p$ as given in (7) into the variational formulation (5), we have to evaluate

$$
\begin{aligned}
\left(\frac{1}{2} I+K_{\kappa}\right) p & =-\rho_{f} \omega^{2}\left(\frac{1}{2} I+K_{\kappa}\right) D_{\kappa}^{-1}\left(\frac{1}{2} I+K_{\kappa}^{\prime}\right)[\mathbf{u} \cdot \mathbf{n}]+\alpha\left(\frac{1}{2} I+K_{\kappa}\right) p_{\kappa^{2}} \\
& =-\rho_{f} \omega^{2}\left(\frac{1}{2} I+K_{\kappa}\right) D_{\kappa}^{-1}\left(\frac{1}{2} I+K_{\kappa}^{\prime}\right)[\mathbf{u} \cdot \mathbf{n}]
\end{aligned}
$$

due to $\operatorname{ker} D_{\kappa}=\operatorname{ker}\left(\frac{1}{2} I+K_{\kappa}\right)$. In fact, the Poincaré-Steklov operator

$$
T_{\kappa}:=V_{\kappa}+\left(\frac{1}{2} I+K_{\kappa}\right) D_{\kappa}^{-1}\left(\frac{1}{2} I+K_{\kappa}^{\prime}\right): H^{-1 / 2}(\Gamma) \rightarrow H^{1 / 2}(\Gamma)
$$

is well defined for all frequencies $\omega$. Hence we conclude the variational problem to find $\mathbf{u} \in\left[H^{1}\left(\Omega_{S}\right)\right]^{3}, \mathbf{u}=\mathbf{g}_{D}$ on $\Gamma_{D}$, such that

$$
\int_{\Omega_{s}}[2 \mu e(\mathbf{u}): e(\mathbf{v})+\lambda \operatorname{div} \mathbf{u} \operatorname{div} \mathbf{v}] d x
$$




$$
-\omega^{2}\left[\rho_{s} \int_{\Omega_{s}} \mathbf{u} \cdot \mathbf{v} d x+\rho_{f}\left\langle T_{\kappa}[\mathbf{u} \cdot \mathbf{n}], \mathbf{v} \cdot \mathbf{n}\right\rangle_{\Gamma}\right]=\int_{\Omega_{s}} \mathbf{f} \cdot \mathbf{v} d x+\int_{\Gamma_{N}} \mathbf{g}_{N} \cdot \mathbf{v} d s_{x}
$$

is satisfied for all $\mathbf{v} \in\left[H^{1}\left(\Omega_{S}\right)\right]^{3}, \mathbf{v}=\mathbf{0}$ on $\Gamma_{D}$. Since the bilinear form which is related to the variational formulation (8) is coercive, injectivity ensures unique solvability of the variational problem $(8)$, see also $[8,9]$.

Theorem 1. Assume that $\omega \in \mathbb{R}$ is not a Jones frequency. Then there exists a unique solution $\mathbf{u}$ of the variational problem (8).

Remark 1. Although boundary value problems of the exterior Helmholtz equation are unique solvable, related boundary integral equations may suffer from spurious modes which correspond to solutions of related interior eigenvalue problems for the Laplacian. Formulations which are stable for all frequencies, are usually based on complex linear combinations of different boundary integral operators, see, e.g., $[2,9]$. However, when using a direct boundary integral approach as presented here, this also leads to a stable formulation, see [14] for a further discussion.

In what follows we consider a frequency $\omega \in \mathbb{R}$ which is not a Jones mode. If the displacement field $\mathbf{u}$ is known as the unique solution of the variational problem (8), we may use the boundary integral equation (6) to determine the pressure $p$. In the case when $\kappa^{2}$ is an eigenvalue of the interior Neumann eigenvalue problem, the solution $p$ as given in (7) is not unique. However, using the transmission conditions (3) we find

$$
p=-T \mathbf{u} \cdot \mathbf{n},
$$

in fact $(\mathbf{u}, p)$ is the unique solution of the coupled variational formulation (5). The representation (9) can be used to modify the boundary integral equation (6) to obtain a formulation which admits a unique solution $p$ for all frequencies, for example we may consider the boundary integral equation

$$
\left[\frac{1}{\rho_{f} \omega^{2}} D_{\kappa}+i \eta \widetilde{D}_{0}\right] p+\left(\frac{1}{2} I+K_{\kappa}^{\prime}\right)[\mathbf{u} \cdot \mathbf{n}]+i \eta \widetilde{D}_{0}(T \mathbf{u} \cdot \mathbf{n})=0 \quad \text { on } \Gamma,
$$

where $\widetilde{D}_{0}$ is the stabilized hypersingular boundary integral operator of the Laplacian [13], and $\eta \in \mathbb{R}$ is some parameter to be chosen. For simplicity of the presentation we only consider the discretization of the variational formulation (8) by using piecewise linear finite elements which are defined with respect to some admissible triangulation of $\Omega_{s}$, and by using piecewise linear boundary elements on $\Gamma$. This leads to the linear system

$$
\left(\begin{array}{cc}
K_{h}^{\mathrm{FEM}}-\omega^{2}\left[\rho_{s} M_{h}^{\mathrm{FEM}}+\rho_{f} N_{h}^{\top} V_{h}^{\mathrm{BEM}} N_{h}\right] N_{h}^{\top}\left(\frac{1}{2} M_{h}^{\mathrm{BEM}}+K_{h}^{\mathrm{BEM}}\right) \\
\left(\frac{1}{2} M_{h}^{\mathrm{BEM}, \top}+K_{h}^{\prime \mathrm{BEM}}\right) N_{h} & \frac{1}{\omega^{2} \rho_{f}} D_{h}^{\mathrm{BEM}}
\end{array}\right)\left(\begin{array}{l}
\underline{u} \\
\underline{p}
\end{array}\right)=\left(\begin{array}{c}
\underline{f} \\
\underline{0}
\end{array}\right) .
$$

Here, $K_{h}^{\mathrm{FEM}}$ and $M_{h}^{\mathrm{FEM}}$ are the finite element stiffness and mass matrices, respectively, and $V_{h}^{\mathrm{BEM}}, M_{h}^{\mathrm{BEM}}, K_{h}^{\mathrm{BEM}}$, and $D_{h}^{\mathrm{BEM}}$ are the Galerkin boundary element matrices, see, e.g., [11], and $N_{h}$ corresponds to the application of the normal component, $\mathbf{u} \cdot \mathbf{n}$. 
From the standard theory, e.g., [13], we expect a second order of convergence when measuring the error $\left\|\mathbf{u}-\mathbf{u}_{h}\right\|_{L_{2}\left(\Omega_{s}\right)}$. Although the pressure $p$ on the boundary $\Gamma$ may not be unique, the computation of the pressure $p$ in $\Omega_{f}$ by means of the exterior representation formula

$$
\widetilde{p}(x)=-\rho_{f} \omega^{2} \int_{\Gamma} U_{\kappa}^{*}(x, y)\left[\mathbf{u}_{h}(y) \cdot \mathbf{n}_{y}\right] d s_{y}+\int_{\Gamma} \frac{\partial}{\partial n_{y}} U_{\mathcal{K}}^{*}(x, y) p_{h}(y) d s_{y} \quad \text { for } x \in \Omega_{f}
$$

is unique, and we conclude a second order convergence of the pointwise error [13].

As a numerical example for the direct simulation we consider the Neumann boundary value problem (1)-(4) with

$$
\Omega_{s}:=\left\{x \in \mathbb{R}^{3}: 0.8<|x|<1\right\}, \quad \Omega_{f}:=\left\{x \in \mathbb{R}^{3}: 1<|x|\right\},
$$

where the exact solution is given by, $r=|x|$,

$$
p(x)=\frac{e^{i \kappa r}}{r} \quad \text { for } r>1, \quad u(r)=\left[c_{1} u_{1}(r)+c_{2} u_{2}(r)\right] e_{r} \quad \text { for } r \in(0.8,1),
$$

and

$$
\begin{aligned}
& u_{1}(r)=-\frac{\sqrt{\lambda+2 \mu} \cos \frac{r \sqrt{\rho_{s}} \omega}{\sqrt{\lambda+2 \mu}}}{r \sqrt{\rho_{s}} \omega}+\frac{(\lambda+2 \mu) \sin \frac{r \sqrt{\rho_{s}} \omega}{\sqrt{\lambda+2 \mu}}}{r^{2} \rho_{s} \omega^{2}}, \\
& u_{2}(r)=-\frac{\sqrt{\lambda+2 \mu} \sin \frac{r \sqrt{\rho_{s}} \omega}{\sqrt{\lambda+2 \mu}}}{r \sqrt{\rho_{s}} \omega}-\frac{(\lambda+2 \mu) \cos \frac{r \sqrt{\rho_{s}} \omega}{\sqrt{\lambda+2 \mu}}}{r^{2} \rho_{s} \omega^{2}} .
\end{aligned}
$$

Note that the constants $c_{1}$ and $c_{2}$ have to be chosen accordingly to satisfy the transmission conditions (3). The material constants are given as $E=105 \cdot 10^{9} \mathrm{~N} / \mathrm{m}^{2}$, $v=0.34$, while the densities of the structure and of the fluid are chosen as $\rho_{s}=1000 \mathrm{~kg} / \mathrm{m}^{3}$ and $\rho_{f}=4500 \mathrm{~kg} / \mathrm{m}^{3}$, respectively. Recall that the speed of sound is $c=1484 \mathrm{~m} / \mathrm{s}$. As frequency we have chosen $\omega=3090 \mathrm{~s}^{-1}$ which corresponds to an eigenfrequency of the hypersingular boundary integral operator $D_{\kappa}$. In Table 1 we present the relative errors of the displacement field both in the $L_{2}(\Omega)$ and in the energy norm, where we observe quadratic and linear convergence, as predicted. In addition, we also give the pointwise error for the pressure which is evaluated in $\widehat{x}=(2,0,0)^{\top}$, again we observe a quadratic convergence as predicted [13].

Table 1 Convergence of the FEM/BEM approach for direct simulation

\begin{tabular}{cccc}
\hline \multirow{2}{*}{$N_{\mathrm{FEM}}$} & $\left\|u-u_{h}\right\|_{L_{2}\left(\Omega_{s}\right)}$ & $\frac{\left\|u-u_{h}\right\|_{H^{1}\left(\Omega_{s}\right)}}{\|u\|_{L_{2}(\Omega)}}$ & $\frac{\|u\|_{H^{1}(\Omega)}}{\|(\widehat{x})-\widetilde{p}(\widehat{x}) \mid}$ \\
\hline 1948 & $9.93-2$ & $2.56-1$ & $5.37-2$ \\
15584 & $2.71-2$ & $1.45-1$ & $1.44-2$ \\
124672 & $7.27-3$ & $7.62-2$ & $3.69-3$ \\
\hline
\end{tabular}




\section{Eigenvalue analysis}

In this section we discuss the solution of the eigenvalue problem which is related to the transmission problem (1)-(4). Based on the coupled formulation (8) of the transmission problem the following related eigenvalue problem is considered: Find $(\omega, \mathbf{u}, p)$ with $(\mathbf{u}, p) \neq(\mathbf{0}, 0)$ such that

$$
A(\omega)\left(\begin{array}{c}
\mathbf{u} \\
p
\end{array}\right):=\left(\begin{array}{cc}
-\omega^{2} \rho_{S} M_{S}+K_{S}-\rho_{f} \omega^{2} N^{*} V_{\kappa} N & N^{*}\left(\frac{1}{2} I+K_{\kappa}\right) \\
\left(\frac{1}{2} I+K_{\kappa}^{\prime}\right) N & \frac{1}{\omega^{2} \rho_{f}} D_{\kappa}
\end{array}\right)\left(\begin{array}{l}
\mathbf{u} \\
p
\end{array}\right)=\left(\begin{array}{l}
\mathbf{0} \\
0
\end{array}\right)
$$

where $M_{S}$ represents the mass term and $K_{S}$ the stiffness term of the structure, and $N \mathbf{u}=\mathbf{u}_{\mid \Gamma} \cdot \mathbf{n}$. The boundary integral operators depend nonlinearly on the wave number $\kappa=\omega / c$, hence (10) is a nonlinear eigenvalue problem in $\omega$. For the eigenvalue problem (10), in addition to the requested eigenvalues we also obtain eigenvalues which correspond to the Laplacian with a Neumann boundary condition. However, in practice the latter can be filtered out very easily.

A Galerkin finite and boundary element discretization of (10) results in a nonlinear matrix eigenvalue problem of the form

$$
A_{h}\left(\omega_{h}\right)\left(\frac{\underline{u}}{\underline{p}}\right)=\left(\frac{\underline{0}}{\underline{0}}\right) .
$$

A rigorous numerical analysis of the Galerkin eigenvalue problem (11) can be carried out within the framework of the concept of eigenvalue problems for holomorphic Fredholm operator-valued functions [15] and will be addressed in a forthcoming paper. This concept provides comprehensive convergence results which include error estimates for the eigenvalues and eigenspaces.

For the numerical solution of (11) we use the contour integral method [1]. This method is suitable for the extraction of all eigenvalues which lie inside of a predefined contour in the complex plane. An alternative approach for the numerical solution of the nonlinear eigenvalue problem (11) which is based on polynomial interpolation is presented in [3].

As a numerical example we consider the Neumann eigenvalue problem for the spherical shell $\Omega_{S}:=\left\{x \in \mathbb{R}^{3}: 4.95<|x|<5\right\}$ and for the fluid domain $\Omega_{f}:=\{x \in$ $\left.\mathbb{R}^{3}:|x|>5\right\}$. For this example analytical approximations of the eigenvalues are derived in [7]. The material constants for the shell are $E=207 \cdot 10^{9} \mathrm{~N} / \mathrm{m}^{2}, v=0.3$ and $\rho_{S}=7669 \mathrm{~kg} / \mathrm{m}^{3}$. For the surrounding fluid, we choose $c=1483.24 \mathrm{~m} / \mathrm{s}$. As ansatz spaces for the Galerkin eigenvalue problem (11) we use piecewise linear finite elements and piecewise linear boundary elements as in the previous section. The eigenvalues of practical interest are those which are lying close to the real axis, since the imaginary part of an eigenvalue corresponds to the damping of the related eigenfunction in time. As domain of interest for the eigenfrequencies $f=\omega /(2 \pi)$ we have chosen the strip $\{f \in \mathbb{C}: 1<\mathfrak{R}(f)<90,-5<\mathfrak{I}(f)<5\}$. In this domain two analytical approximations are given in [7]. The results of the contour integral method are presented in Table 2 for different meshes. The approximations of the 
eigenvalues on the two finest mesh levels match well with the analytical approximations.

Table 2 Approximations of the two smallest non-zero eigenvalues $f=\omega /(2 \pi)$

\begin{tabular}{|c|c|c|c|}
\hline$h /$ dof $0.5 / 8794$ & $0.25 / 36792$ & $0.15 / 109455$ & anal. approx. \\
\hline $\begin{array}{l}(58.19,-1.44) \\
(58.26,-1.45) \\
(58.50,-1.48) \\
(58.62,-1.50) \\
(58.96,-1.54)\end{array}$ & $\begin{array}{r}(55.82,-1.18) \\
(55.84,-1.18) \\
(55.84,-1.18) \\
(56.03,-1.20) \\
(56.04,-1.21)\end{array}$ & $\begin{array}{l}(55.65,-1.16) \\
(55.66,-1.16) \\
(55.66,-1.16) \\
(55.78,-1.18) \\
(55.78,-1.18)\end{array}$ & 56.02 \\
\hline $\begin{array}{l}(83.61,-1.00) \\
(83.73,-1.03) \\
(84.51,-1.08) \\
(85.10,-1.14) \\
(85.47,-1.16) \\
(85.94,-1.18) \\
(87.96,-1.37)\end{array}$ & $\begin{array}{r}(71.47,-0.32) \\
(71.53,-0.32) \\
(71.63,-0.32) \\
(71.63,-0.32) \\
(71.72,-0.33) \\
(71.74,-0.33) \\
(71.80,-0.34)\end{array}$ & $\begin{array}{l}(70.45,-0.31) \\
(70.53,-0.31) \\
(70.53,-0.31) \\
(70.54,-0.31) \\
(70.60,-0.31) \\
(70.61,-0.31) \\
(70.62,-0.32)\end{array}$ & 70.52 \\
\hline
\end{tabular}

\section{Conclusions}

The symmetric formulation of finite and boundary element methods for vibroacoustic interface problems turns out to be stable for almost all freqencies. If we exclude Jones frequencies, no spurious modes appear. In fact, we can avoid the use of combined boundary integral equation formulations such as Brakhage/Werner and Burton/Miller, see, e.g., [2, 14], which require sufficient smoothness of the coupling interface. For the acceleration of the numerical simulations one may use fast boundary element methods such as the adaptive cross approximation [11] or the fast multipole method [2]. In addition, the design of appropriate preconditioned iterative solvers is a challenging task not only for the direct simulation. In fact, the contour integral method allows an reliable and accurate computation of eigenvalues within a given domain of interest, without any knowledge on the number and on the position of eigenvalues. Applications of the proposed methodologies include the simulation and eigenvalue analysis of ships, see Fig. 2 for a simplified model of a submarine made of titanium. The length is $12 m$, its diameter $2 m$, and its wall thickness $0.1 \mathrm{~m}$. The first eigenfrequency is $f=52.12-0.007 i$, the related eigensolution is given in Fig. 2. This simulation was done by using 67.145 tetrahedral finite elements and 17.372 triangular boundary elements, which results in 74.523 global degrees of freedom. 
Fig. 2 Real and imaginary part of an eigensolution of a simplified submarine
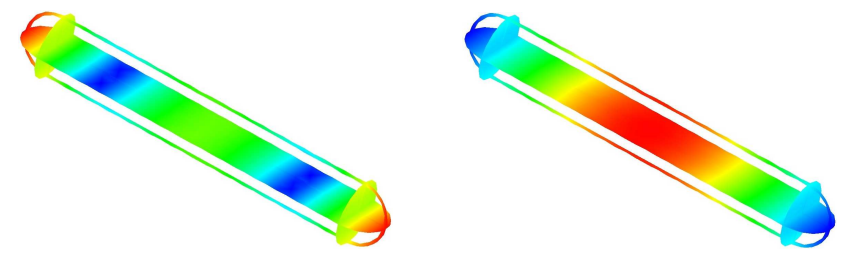

\section{References}

1. Beyn, W.J.: An integral method for solving nonlinear eigenvalue problems. Linear Algebra Appl. 436, 3839-3863 (2012)

2. Brunner, D., Of, G., Junge, M., Steinbach, O., Gaul, L.: A fast BE-FE coupling scheme for partly immersed bodies. Int. J. Numer. Meth. Engrg. 81, 28-47 (2010)

3. Effenberger, C., Kressner, D., Steinbach, O., Unger, G.: Interpolation-based solution of a nonlinear eigenvalue problem in fluid-structure interaction. PAMM 12, 633-634 (2012)

4. Hsiao, G.C., Kleinman, R.E., Roach, G.F.: Weak solutions of fluid-solid interaction problems. Math. Nachr. 218, 139-163 (2000)

5. Ihlenburg, F.: Finite element analysis of acoustic scattering. Springer (1998)

6. Jones, D.S.: Low-frequency scattering by a body in lubricated contact. Quart. J. Mech. Appl. Math. 36, 111-138 (1983)

7. Junger, M., Feit, D.: Sound and Structures and their Interaction. MIT Press, Cambridge (1986)

8. Kimeswenger, A., Steinbach, O.: Symmetric BEM/FEM coupling for vibro-acoustic fluidstructure interaction problems (2013, in preparation)

9. Luke, C.J., Martin, P.A.: Fluid-solid interaction: acoustic scattering by a smooth elastic obstacle. SIAM J. Appl. Math. 55, 904-922 (1995)

10. Of, G., Steinbach, O.: Coupled FE/BE formulations for the fluid-structure interaction. In: Domain Decomposition Methods in Science and Engineering XIX, Lecture Notes in Computational Science and Engineering, vol. 78, pp. 293-300. Springer, Heidelberg (2011)

11. Rjasanow, S., Steinbach, O.: The fast solution of boundary integral equations. Springer, New York (2007)

12. Schwarze, G.: Über die 1., 2. und 3. äussere Randwertaufgabe der Schwingungsgleichung. Math. Nachr. 28, 337-363 (1965)

13. Steinbach, O.: Numerical approximation methods for elliptic boundary value problems. Finite and boundary elements. Springer, New York (2008)

14. Steinbach, O.: Boundary integral equations for Helmholtz boundary value and transmission problems. In: Direct and inverse problems in wave propagation and applications, Radon Series on Computational and Applied Mathematics, vol. 14, pp. 253-292. de Gruyter, Berlin (2013)

15. Steinbach, O., Unger, G.: Convergence analysis of a Galerkin boundary element method for the Dirichlet Laplacian eigenvalue problem. SIAM J. Numer. Anal. 50, 710-728 (2012) 